\title{
Atmospheric Parameters and Chemical Abundances for Herbig Ae stars $\dagger$
}

\author{
Bruno V. Castilho ${ }^{1}$, Simone Daflon ${ }^{2}$, Marília J. Sartori ${ }^{1}$, \\ and Norbert Przybilla ${ }^{3}$ \\ ${ }^{1}$ Laboratório Nacional de Astrofísica/MCT \\ Rua Estados Unidos, 154 - 37504-364 - Itajubá, MG, Brazil \\ email: bruno@lna.br marilia@lna.br \\ ${ }^{2}$ Observatório Nacional/MCT \\ Rua Gal. José Cristino, 77 - 20921-400 - Rio de Janeiro, RJ, Brazil \\ email: daflon@on.br \\ ${ }^{3}$ Dr. Remeis-Sterwarte Bamberg, Universität Erlangen-Nürnberg \\ Sternwartstr. 7, D-96049 Bamberg, Germany \\ email: przybilla@sternwarte.uni-erlangen.de
}

\begin{abstract}
In this work, we present temperature, surface gravity, metallicity, microturbulence and element abundances, determined from a detailed spectroscopic analysis for a sample of 9 Herbig Ae stars, based on high resolution, high S/N spectra.
\end{abstract}

Keywords. stars: abundances, stars: pre-main-sequence

\section{Introduction}

The study of the chemical composition is an important way to understand the evolution of stars. In the case of pre-main-sequence (PMS) stars these studies are even more necessary since there is still few data available in the literature. Furthermore, the study of the chemical composition of intermediate mass PMS stars (Herbig Ae/Be stars) may contribute to understand the relationship between the frequency of planets and stellar metallicity. In this work we present the stellar parameters and abundance analysis of a sample of 9 Herbig Ae stars based on spectra obtained with the FEROS spectrograph (ESO).

\section{Analysis}

Effective temperatures could not be determined using photometric calibrations for these stars, because the circumstellar reddening is unknown. The preliminary determination of the temperature and gravity are obtained through the spectral classification, based on types determined by Torres (1999) and the calibration by Gray \& Corbally (1994). These values are then refined by fitting theoretical $\mathrm{H} \gamma$ profiles from Kurucz (1993) to the observed spectra.

For stars with enough measurable FeI lines in their spectra the excitation equilibrium was used to refine the temperature. The gravity was estimated by the FeI/FeII ionization equilibrium. Metallicity was determined through the curves of growth of Fe I and II. The curves were calculated with the code Renoir (by M. Spite) and using the same atmospheric models as the $\mathrm{H} \gamma$ fitting. The $T_{\text {eff }}$ values derived from Fe lines agree very well with the $T_{\text {eff }}$ obtained from the fitting of $\mathrm{H} \gamma$ profiles $(<T(\mathrm{H} \gamma)-T(\mathrm{Fe})>=49 \pm 111 K)$.

$\dagger$ Based on observations collected at the European Southern Observatory, Chile. 
Table 1. Stellar parameters and abundances determined for the sample stars.

\begin{tabular}{lcccccccccc}
\hline $\begin{array}{l}\text { Star } \\
\text { HD }\end{array}$ & $\begin{array}{l}T_{\text {eff }} \\
{[K]}\end{array}$ & $\log g$ & {$[\mathrm{Fe} / \mathrm{H}]$} & $\log \epsilon(\mathrm{C})$ & $\begin{array}{c}\log \epsilon(\mathrm{O}) \\
\text { NLTE }\end{array}$ & $\log \epsilon(\mathrm{N})$ & $\log \epsilon(\mathrm{S})$ & $\log \epsilon(\mathrm{Ca})$ & $\log \epsilon(\mathrm{Ti})$ & $\log \epsilon(\mathrm{Sr})$ \\
\hline 101412 & 10000 & 4.3 & -0.05 & 9.04 & 8.66 & 8.84 & 7.81 & 6.5 & 4.31 & 2.52 \\
142666 & 7100 & 4.0 & 0.00 & 8.5 & 8.72 & 9.57 & 7.18 & 6.09 & 5.09 & \\
144432 & 7250 & 4.0 & 0.10 & 8.3 & 8.68 & 9.86 & 7.25 & 6.29 & 4.79 & \\
145718 & 7500 & 3.5 & 0.20 & 8.3 & 8.59 & & 7.32 & 6.3 & 4.89 & \\
100453 & 7150 & 4.0 & 0.00 & 8.25 & 8.7 & 8.85 & 7.25 & 5.95 & 4.67 & 3.08 \\
139614 & 7700 & 4.5 & -0.20 & 8.4 & 8.67 & 8.23 & 6.94 & 6.02 & 5.06 & \\
141569 & 10000 & 4.2 & 0.00 & 8.5 & 8.71 & & & & & \\
163296 & 8500 & 4.0 & 0.00 & 8.2 & 8.75 & & & 5.11 & 5.02 & \\
169142 & 7400 & 4.5 & -0.30 & 8.55 & 8.62 & 8.87 & & 5.69 & 4.49 & 2.81 \\
\hline
\end{tabular}

Abundance analysis was based on the fully-blanketed and plane-parallel LTE model atmospheres calculated with the ATLAS9 code (Kurucz 1993) for a constant microturbulent velocity of $2 \mathrm{~km} / \mathrm{s}$ and solar composition. LTE abundances were determined by fitting synthetic spectra calculated with program LINFOR (originally developed by $\mathrm{H}$. Holweger, M. Steffen, and W. Steenbock) to the selected spectral regions. The theoretical profiles were broadened to account for effects of rotation, limb darkening and instrumental profile. CI lines yield carbon solar abundance $\left(\log \epsilon(\mathrm{C})_{\odot}=8.41 \pm 0.03\right.$, Asplund 2003). The mean oxygen abundance derived is 0.2 dex higher than in the $\operatorname{Sun}(\log \epsilon(\mathrm{O}) \odot=8.66 \pm 0.03$, Asplund 2003) and should be analyzed with caution. In an independent determination $\log \epsilon(\mathrm{O})$ departures from LTE were considered in the line formation calculations with the newest version of the program DETAIL (Giddings 1981). The adopted oxygen model atom is described in Przybilla et al. (2000). The synthetic line profiles were calculated with the SURFACE code (Butler \& Giddings 1985), assuming Voigt profile functions. These profiles were then broadened by means of convolution with the rotational profile, including $v \sin i$, limb darkening, and instrumental profile.

\section{Conclusions and next steps}

Our results show that, except for HD169142, the $[\mathrm{Fe} / \mathrm{H}]$ values agree with the Solar metallicity within one sigma. The observed abundance pattern, however, may be related to the star-forming regions associated with these objects. The LTE elemental abundances $[\mathrm{X} / \mathrm{H}]$ derived for $\mathrm{Ca}, \mathrm{S}, \mathrm{Ti}$ and $\mathrm{Sr}$ are in average 0.20 dex lower than the Sun, with some individual abundances close to the solar values. Determination of NLTE abundances of $\mathrm{C}$ and $\mathrm{N}$ by spectral synthesis is in progress for the present sample.

\section{Acknowledgements}

The authors would like to thank LNA/MCT, CNPq and FAPEMIG for financial support to attend the meeting.

\section{References}

Asplund, M. 2003, in: C. Charbonnel, D. Schaerer, \& G. Meynet (eds.), CNO in the Universe, ASP Conf. Series Vol. 304 (San Francisco: ASP), p. 275

Butler, K. \& Giddings, J. R. 1985, Newsletter on Analysis of Astronomical Spectra, No. 9 (London: Univ. London)

Giddings, J. R. 1981, Ph.D. Thesis, University of London

Gray, R. O. \& Corbally, C. J. 1994, AJ, 107, 742

Kurucz, R. L. 1993, ATLAS9 Stellar Atmosphere Programs and $2 \mathrm{~km} / \mathrm{s}$ grid, CD-ROM No.13 (Cambridge, Mass.: Smithsonian Astrophysical Observatory)

Przybilla, N., Butler, K., Becker, S. R., Kudritzki, R. P., \& Venn, K. A. 2000, A\&A, 359, 1085

Torres, C. A. O. 1999, Ph.D. Thesis, Pub. Esp. 10, Observatório Nacional, Brasil 